\title{
Lydie BODIOU, Véronique MEHL, Jacques OULHEN,
} Francis PROST et Jérôme WILGAUX (dir.) Chemin faisant. Mythes, cultes et société en Grèce ancienne. Mélanges en l'honneur de Pierre Brulé

Rennes, Presses universitaires de Rennes, 2009, coll. « Histoire ancienne ", $314 \mathrm{p}$.

\section{Charles Delattre}

\section{(2) OpenEdition}

\section{Édition électronique}

URL : http://journals.openedition.org/clio/10924

DOI : 10.4000/clio.10924

ISSN : 1777-5299

\section{Éditeur}

Belin

\section{Édition imprimée}

Date de publication : 31 décembre 2012

ISSN : 1252-7017

\section{Référence électronique}

Charles Delattre, «Lydie bodıou, Véronique meHL, Jacques oulhen, Francis prost et Jérôme wiLgaux (dir.) Chemin faisant. Mythes, cultes et société en Grèce ancienne. Mélanges en I'honneur de Pierre Brulé ", Clio. Femmes, Genre, Histoire [En ligne], 36 | 2012, mis en ligne le 23 août 2013, consulté le 22 septembre 2020. URL : http://journals.openedition.org/clio/10924 ; DOl : https://doi.org/10.4000/clio.10924

Ce document a été généré automatiquement le 22 septembre 2020.

Tous droits réservés 


\section{Lydie BODIOU, Véronique MEHL,} Jacques OULHEN, Francis PROST et Jérôme WILGaUX (dir.) Chemin faisant. Mythes, cultes et société en Grèce ancienne. Mélanges en l'honneur de Pierre Brulé

Rennes, Presses universitaires de Rennes, 2009, coll. « Histoire ancienne ", $314 \mathrm{p}$.

Charles Delattre

\section{RÉFÉRENCE}

Lydie BODIOU, Véronique MEHL, Jacques OULHEN, Francis PRost et Jérôme WILGAUX (dir.), Chemin faisant. Mythes, cultes et société en Grèce ancienne. Mélanges en l'honneur de Pierre Brulé, Rennes, Presses universitaires de Rennes, 2009, coll. « Histoire ancienne », 314 p.

1 Le volume Chemin faisant réunit vingt-et-un articles rassemblés en hommage à l'historien de la Grèce classique Pierre Brulé et aux activités qu'il a développées au sein du CRESCAM-LAHM (UMR 6566). Il peut ainsi servir de pendant à La Grèce d'à côté, un recueil d'articles de P.Brulé publié en 2007: les compagnonnages de l'historien, illustrés par la participation de P. Briant, de M.-M. Mactoux, de L. Bruit-Zaidman, de R. Parker, de S. Georgoudi, de M. Jost, de V. Dasen ou d'A. Powell, pour n'en citer que quelques-uns, dessinent une constellation intellectuelle passionnante.

2 Les perspectives de recherche assumées dans le volume sont celles-là même du destinataire de l'hommage $:$ histoire des femmes, histoire religieuse et histoire du corps 
constituent un triptyque qui montre la diversité des intérêts de P. Brulé. Le volume illustre également la faculté que P. Brulé a eue d'entretenir un compagnonnage amical avec ses collègues: quoique l'auteur de ce compte-rendu ne le connaisse pas personnellement, il a été sensible aux détails surprenants et pleins d'humour présentés dans l'abécédaire et l'introduction, détails qui donnent une image très sympathique des habitudes matinales de P. Brulé, de son passé d'élève de lycée agricole à Saintes ou de son statut de "paysan contrarié" (p. 13). On ne cherchera pas dans le volume une unité autre que celle qui renvoie à $\mathrm{P}$. Brulé lui-même, à ses objets de recherche et à ses cheminements (d'où le titre) : à cet égard, lui seul était peut-être capable de rendre compte avec justesse de cet ensemble foisonnant. On se contentera ici d'indiquer le thème de chacun des articles en analysant les grandes lignes de ceux qui concernent plus particulièrement l'histoire des femmes et du genre.

3 La première partie constitue une "mise en route" qui reprend les premiers champs d'étude de P. Brulé, ce qui explique son manque d'unité, mais ne remet pas en cause son intérêt. Pierre Briant ouvre le bal en montrant comment l'historiographie occidentale moderne sur le monde perse dépend de l'image d'Alexandre, de ses conquêtes et de son empire, et s'attache au cas particulier du XVIII ${ }^{e}$ siècle, où se développe une vision orientaliste qui postule la décadence de l'empire perse, à la suite de Bossuet et de Rollin. Stéphanie Maillot propose une étude sur une association de sculpteurs rhodiens au $\mathrm{II}^{\mathrm{e}} \mathrm{s}$. avant notre ère, le koinon des Asklapiastai Olympiastai Nikasioneioi. Marie-Madeleine Mactoux s'intéresse au fouet et à l'usage qu'on en faisait sur les esclaves. Pour elle, le châtiment du fouet est constitutif de l'esclave, non seulement comme pratique sociale permettant le châtiment et affermissant le pouvoir du maître, mais aussi pour dire l'esclave, qui peut en prendre le nom (mastigias). Enfin, Anton Powell propose une synthèse sur l'histoire de Sparte et la fameuse "révolution" qui l'aurait transformée de fond en comble. Partant de l'idée que les jugements antiques sur Sparte sont idéalisants, l'auteur propose de renverser ces idéaux pour identifier la peur qu'ils conjurent. Mollesse, faiblesse, aveu de l'échec, diversité et désunion sont les peurs de l'âge classique. Par contraste, les coupes laconiennes des années 580-530 montrent les plaisirs d'une société aristocratique non soumise à l'idéal. D'une époque à l'autre, Sparte a connu une évolution rapide, où l'austérité garde le souvenir inversé de ce qui l'a précédée, tout en niant que cela ait jamais existé.

4 Laurent Piolot inaugure une deuxième partie, "Chemins de femmes", et étudie les gynéconomes, magistrats connus à partir de la fin du IV $\mathrm{e}$. (47 occurrences de natures diverses). Leur sphère d'action est celle des affaires communes (koinon), non du particulier (idion). Ils veillent à l'eukosmia des femmes, c'est-à-dire des femmes "de bonnes mœurs" (une expression curieuse qui revient p. 112 et 113). Mais une relecture prudente de l'inscription des mystères d'Andanie $(\$ 4,1.15-26)$ montre qu'ils pouvaient exercer leur surveillance aussi sur les hommes, ce qui est confirmé par d'autres sources. Les gynéconomes, qui ont peut-être été institués par Lycurgue à Athènes entre 336 et 324 (L. Piolot adopte ici l'hypothèse de B. Garland), s'occupaient des femmes à l'occasion de leurs sorties, fêtes de la cité et cérémonies de deuil, ils n'étaient pas une police des mœurs.

5 Louise Bruit-Zaidman poursuit l'enquête commencée par N. Ernoult sur la place des femmes dans la législation de la cité des Magnètes en envisageant la part qui leur est réservée non seulement dans l'éducation ou la vie sociale, mais aussi dans le domaine religieux, à partir d'une lecture du livre VIII des Lois de Platon. Là comme ailleurs, pas 
de distinction marquée entre hommes et femmes, "citoyens" et "citoyennes", les individus s'insérant dans des organisations qui sont soit mixtes, soit composées en parallèle. Non seulement les prêtrises sont largement étendues aux femmes, mais elles occupent aussi la fonction d'inspectrices des mariages. Cependant leur citoyenneté n'est pas identique à celle des hommes, car elle n'est pas politique, même si elles participent activement à la vie de la polis. Ce biais est lié à la reconnaissance, affirmée par Platon, d'une différence de nature, qui nécessite la surveillance particulière des femmes, y compris par elles-mêmes.

6 Anne Jacquemin s'intéresse à la présence des femmes à Olympie: si l'exclusion des femmes mariées est certaine, on pouvait compter sur la présence indispensable de certaines prêtresses, sans oublier la célébration en parallèle des Heraia par des femmes. Les femmes d'Élis jouaient même un rôle particulier dans la célébration olympique en entonnant un chant de déploration en l'honneur d'Achille : elles assuraient rien moins que l'ouverture du culte de Zeus aux Olympiades. Achille sortait alors de son rôle traditionnel pour jouer le rôle d'un agonothète.

7 Karine Karila-Cohen propose une étude prosopographique très riche sur les canéphores aux Pythaïdes, théorie envoyée par Athènes à Delphes, à la fin de l'époque hellénistique, à intervalles réguliers. Claudine Leduc propose ensuite de reprendre le dossier de l'énigmatique Kourotrophos de l'Acropole, déesse sans théonyme, en comparant ce dossier et en l'associant à celui de l'olivier d'Athéna, arbre sacré sans culte, et surtout arbre féminin dit paidotrophos. Le contexte général dans lequel Kourotrophos et arbre sacré interviennent est celui d'une constellation religieuse autour d'Athéna Polias : l'une et l'autre permettent de définir un point fixe à partir duquel se définissait une citoyenneté avant les réformes soloniennes. S'ils assuraient des fonctions différentes dans la pratique, ils montraient un même intérêt pour le maillage et la cohésion du territoire. C. Leduc conclut sur la tentation que l'on peut éprouver à identifier l'une à l'autre: la Kourotrophos ne pourrait-elle pas être conçue comme la "représentation animée" de l'olivier?

8 L'article de C. Leduc offre une transition vers la troisième partie, intitulée "Vers le polythéisme". Cette troisième partie est inaugurée par Robert Parker, qui examine, dans la ligne d'une révision générale des éléments fondamentaux du sacrifice (vocabulaire, pratiques, idéologie), le problème de l'identité du "sacrifiant". L'opposition que l'on peut faire entre cette fonction et celle de "sacrificateur" est en effet utile, mais correspond à une distinction non opératoire dans la langue grecque. Revenant sur les généalogies de Pan, en particulier sur la version répandue à l'époque hellénistique et romaine qui en fait le fils d'Hermès et de Pénélope, Madeleine Jost en étudie les rapports avec les traditions arcadiennes, en particulier celles de Mantinée, du mont Lycée et de Tégée. Quant à Paolo Scarpi, il propose une réflexion générale, trop générale peut-être dans un cadre aussi réduit, entre action rituelle initiatique et récit mythique, en relation à la fois avec la figure du héros (ici unilatéralement confondu avec la catégorie des demi-dieux) et avec le passage d'une échelle locale à une échelle panhellénique.

9 Sylvain Lebreton revient sur l'épiclèse Omèstès, attribuée par Plutarque à Dionysos dans deux passages (Vie de Thémistocle, 13, et Vie d'Antoine, 24). Contrairement à ceux qui tirent des deux textes de Plutarque l'idée que cette épiclèse était cultuellement active à Athènes et à Éphèse, S. Lebreton réserve à Lesbos seule l'existence d'un Dionysos Omèstès. Le problème des épiclèses, dont on sait combien il est important dans les 
activités du CRESCAM, fournit également à Corinne Bonnet l'occasion de s'intéresser au monde religieux phénicien. Les épiclèses y apparaissent, non comme le signe d'un ancrage dans le territoire, mais comme une possibilité d'interroger les rapports masculin / féminin dans le panthéon, en particulier la difficile définition de la parèdre du dieu suprême, Astarté. "Nom de Baal", la déesse est associée directement à la puissance du dieu et est une force agissante. Quant à la Tanit nord-africaine, elle est "face de Baal", ce qui lui confère un pouvoir similaire. Ainsi, connaître le nom et voir le visage, partager ce nom et ce visage, organisent un réseau de connivences, qui non seulement assure la cohésion du couple divin, mais fait de la déesse un intercesseur auprès de la puissance divine suprême.

Stella Georgoudi conclut la partie du volume consacrée aux questions cultuelles en revenant sur deux expressions modernes, celles de "trépied prophétique" et de "laurier prophétique", que l'on associe à Apollon dans sa fonction mantique. Elle combat en particulier l'idée, nombreuses sources à l'appui, que trépied et laurier aient été les instruments privilégiés de l'oracle. Ce n'est que par métonymie poétique, dans le monde latin ou chez Nonnos, que le trépied prend voix.

11 Six articles composent la dernière partie du volume, "Itinéraires corporels". Dans le premier d'entre eux, Hélène Bectarte s'intéresse aux stèles funéraires athéniennes de l'époque classique et s'oppose à la doxa qui postule une indifférenciation générale ou une neutralité des vêtements, du point de vue en particulier des classes sociales. Elle montre, à partir d'une étude statistique très précise, comment le costume de l'épouse renseigne sur son identité (de genre) et son statut (d'épouse), en la différenciant à la fois de l'ensemble des hommes et des autres femmes. L'auteur note aussi (sans insister cependant sur la question du réalisme des représentations) que le costume porté sur les stèles implique souvent un voilement partiel du corps féminin, alors que la scène renvoie à l'intérieur domestique: ce serait le signe d'une tension entre sphère publique, celle de la fonction mémorielle des stèles, et privée, celle du contexte représenté.

Rachel Touzé s'attaque à un riche dossier, celui du myrte, dont elle montre à la fois les associations avec la sphère traditionnellement associée à Aphrodite (mais pourquoi en exclure la guerre, dont on connaît les affinités avec la déesse depuis les travaux de G. Pironti ?), et la diversité des emplois, en particulier en lien avec d'autres divinités (Dionysos, Déméter et Coré, Apollon etc.). Les textes botaniques et médicaux sont largement sollicités, et mériteraient d'être encore davantage exploités : l'astringence du mythe, bien notée par R. Touzé, pourrait inciter à des analyses se fondant par exemple sur les réflexions de J. Scarborough sur la dunamis (et non la phusis) des plantes $^{1}$, en plus des rapprochements suggestifs opérés par l'auteur de l'article.

13 L'article de Gaucelm Boismenu propose une exploration d'une Athéna poussée au paradoxe, une Athéna parthenos traitée comme une adolescente par l'auteur de l'article, mais aussi, assure-t-il, par Homère. Un certain nombre d'observations sonnent juste et permettent de relire des passages de l'odyssée d'un œil nouveau. Mais l'appareillage théorique est parfois discutable, en particulier quand l'auteur renvoie sans autre forme de procès les scènes de l'odyssée à la situation réelle de l'oikos archaïque, les personnages à une forme de psychologie des relations familiales (Athéna, fille sans mère), ou la structure textuelle à une conception de l'épopée comme "roman" (p. 261, passim). 
14 Véronique Dasen s'intéresse au corpus des amulettes, plus précisément à celles en pierre semi-précieuse (cornaline, jaspe) qui, sous le Haut Empire, ont été fréquemment portées par les femmes et qui se rapportaient aux événements de leur "vie génitale", menstruation, grossesse et accouchement. Elle étudie en particulier une cornaline de la collection Derek J. Content qui représente une "Baubô", figure bien connue sur les terres cuites de l'Égypte romaine. Quoiqu'on n'ait pas conservé ce type d'amulette à l'époque classique en Grèce, plusieurs textes font explicitement allusion à leur port, qui est confirmé par quelques vases où se voient des femmes nues, courtisanes ou femmes et filles de citoyen, portant attachés au bras ou à la jambe de petits objets où l'auteur propose de reconnaître des amulettes favorisant la bonne santé des femmes.

Gwenaëlle Le Person revient sur l'idée moderne que la médecine hippocratique serait fondamentalement rationnelle et s'opposerait aux pratiques magiques, une idée que certains spécialistes défendent en s'appuyant en particulier sur les premiers paragraphes du traité Maladie sacrée, qui semble aller dans ce sens. Elle souligne avec justesse le caractère polémique du texte, ainsi que les exagérations ou les non-dits de l'auteur du traité. L'auteur retrouve dans le discours de vérité promu par Eusèbe les caractéristiques des relations de pouvoir identifiées par M. Foucault comme "biopouvoir" (un terme que Foucault réserve plutôt aux sociétés normatives contemporaines), ce qui ne facilite pas forcément l'explicitation de son analyse ni l'exploitation de son corpus.

16 La diversité des thèmes abordés, la précision et la finesse des analyses, le désir même du lecteur de voir certaines démonstrations développées et poursuivies, rendent un juste hommage à P. Brulé, par le biais d'un ouvrage que tout rayonnage consacré à l'histoire ancienne se doit d'acquérir.

\section{NOTES}

1. J. Scarborough, "The Pharmacology of Sacred Plants, Herbs, and Roots", in C.A. Faraone, D. Obbink (dir.), Magika Hiera, 1991, p. 139-174.

\section{AUTEURS}

\section{CHARLES DELATTRE}

Université Paris Ouest Nanterre, THEMAM, UMR 7041 ArScAn 